\title{
LONG-PERIOD VARIABLES IN THE MAGELLANIC CLOUDS
}

\author{
P.R. WOOD ${ }^{1}$, G.K.G. MOORE 2 and S.M.G. HUGHES ${ }^{3}$ \\ 1 Mount Stromlo and Siding Spring Observatories, Private Bag, \\ Weston Creek PO, Canberra ACT 2611, Australia

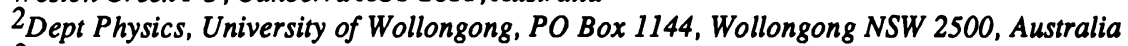 \\ 3 Anglo-Australian Observatory, Private Bag, Coonabarabran NSW 2357, Australia
}

\section{Surveys for long-period variables in the Magellanic Clouds}

Until recently, surveys for variable stars in the Magellanic Clouds were carried out in blue and visual passbands, but these passbands are not ideal for finding very cool stars such as long-period variables (LPVs). Consequently, the early surveys generally found only the brightest LPVs in the Magellanic Clouds. The most extensive surveys were the Harvard surveys whose results are summarized by Payne-Gaposhkin and Gaposhkin 1966 (SMC) and Payne-Gaposhkin 1971 (LMC), with some supplementary data in Hodge and Wright (1969) and Wright and Hodge (1971). LPVs were also found during other smaller surveys such as those of Dessy (1959), Butler $(1972,1978)$ and Lloyd Evans (1971, see also Glass and Lloyd Evans 1981, Lloyd Evans 1978, 1985 and Lloyd Evans et al. 1988).

The surveys by Lloyd Evans were actually aimed at finding LPVs and, because of the very red nature of the LPVs, Lloyd Evans used I as well as V plates in his searches. Recently, extensive surveys for LPVs in the Magellanic Clouds using I plates from the UK Schmidt telescope have now been carried out; these surveys were initiated by Dr. B.L. Turtle. Results obtained from these surveys in the LMC have been published by Wood et al. (1985), Glass and Reid (1985), Reid et al. (1988) and Hughes (1989); results for the SMC have been obtained by Moore (1990).

With the advent of the IRAS satellite, many point sources were found in the Magellanic Clouds. Some of the most luminous optically visible LPVs (including objects found on I plates) were detected by IRAS, but many of the IRAS point sources have no optical counterparts. The colours of some of these IRAS sources indicate that they may be variable $\mathrm{OH} / \mathrm{IR}$ stars, and a number of them have been monitored in the near infrared (JHK); some variable sources have been found by Whitelock et al. (1989) in the SMC and by Wood et al. (1990) in the LMC and SMC.

\section{Infrared photometry}

To derive useful quantities such as $\mathrm{M}_{\text {bol }}$ and $\mathrm{T}_{\text {eff }}$ (estimates), infrared JHK photometry is required for the LPVs. Such photometry can be found in papers by Glass (1979), Feast et al. (1980), Catchpole and Feast (1981), Glass and Feast (1982b), Wood et al. (1983), Frogel (1983), Wood et al. (1985), Glass and Reid (1985), Elias et al. (1985), Wood and Bessell (1985), Bessell et al. 1986), Lloyd Evans et al. (1988), Reid et al.(1988), Whitelock et al. (1989), Feast et al. (1989) and Hughes and Wood (1990). 


\section{Physical properties of LPVs}

In Fig1, a large sample LMC LPVs with spectral types of M or MS and with well-determined periods and $\mathrm{JHK}\left(\mathrm{L}^{\prime}\right)$ photometry (for determining $\mathrm{M}_{\mathrm{bol}}$ ) is plotted. Means of the photometry are used for stars with multiple observations. As shown by Wood et al. (1983), the LPVs can be divided into two distinct groups - the core helium burning supergiants, which have small pulsation amplitudes (0.2-0.3 mag.), and the AGB stars which have larger amplitudes (typically 0.7 mag.) and which frequently exhibit signs of dredge-up at helium shell flashes (i.e. spectra of type MS or C) as expected for evolved AGB stars.

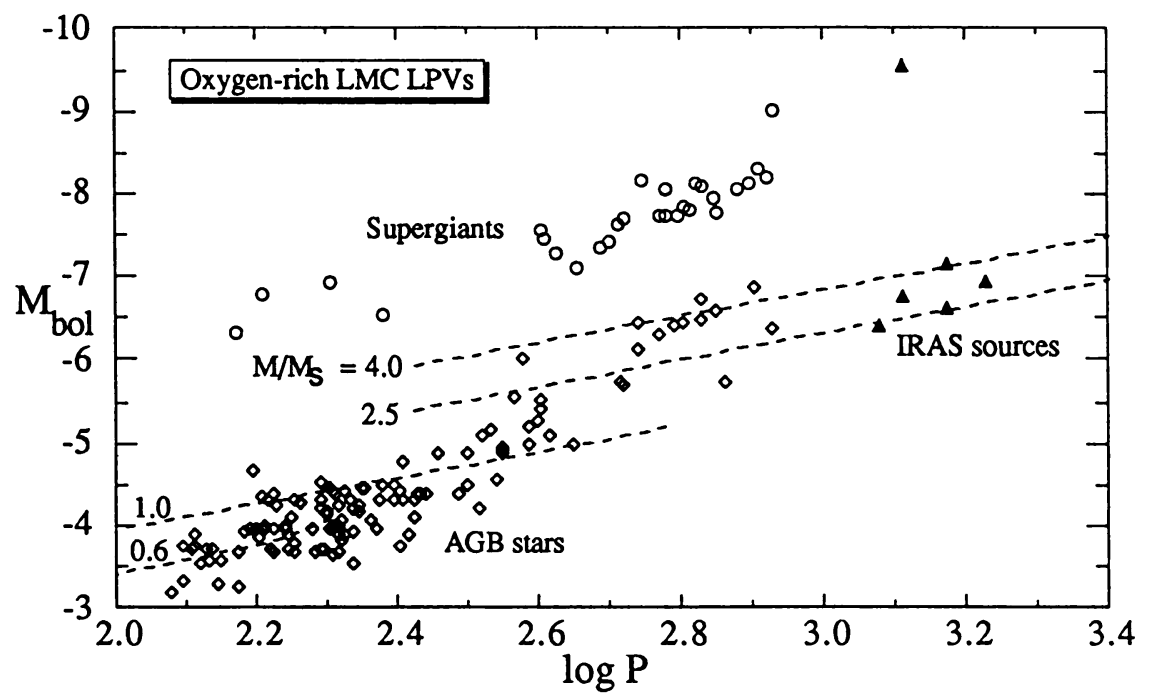

Figure 1. A sample of oxygen-rich LPVs in the LMC which have well-determined periods and JHK $\left(L^{\prime}\right)$ photometry. Open circles are supergiants, filled triangles are IRAS sources (all but one are $\mathrm{OH} / \mathrm{IR}$ stars), and open diamonds are AGB stars. The lines of constant pulsation mass are derived from the formulae of Wood (1989) with a metal abundance $\mathrm{Z}=\mathrm{Z}_{\mathrm{O}} / 2$ for $\mathrm{M} \geq 2.5 \mathrm{M}_{\mathrm{O}}$ and $\mathrm{Z}=\mathrm{Z}_{\mathrm{O}} / 4$ for $\mathrm{M} \leq 1.0 \mathrm{M}_{\mathrm{O}}$.

The new feature shown in Fig 1 is the group of IRAS sources, all but one of which are $\mathrm{OH} / \mathrm{IR}$ stars (i.e. they have $1612 \mathrm{MHz}$ maser emission). There is one OH/IR star whose luminosity $\left(\mathrm{M}_{\mathrm{bol}}<-9\right)$ means that it is clearly a supergiant; its $\mathrm{K}$ amplitude is also small ( 0.2 mag.) as expected for a supergiant. The other five IRAS sources shown are almost certainly AGB stars; the pulsational amplitudes at $\mathrm{K}$ (typically 2 mag. for this group) are large, as found for other AGB pulsators, and the bolometric luminosities put them below the AGB limit of $\mathrm{M}_{\mathrm{bol}} \approx-7.1$.

These stars seem to have evolved at a more-or-less constant luminosity from the group of optically visible upper AGB LPVs with $\mathrm{M}_{\mathrm{bol}} \sim-6$ to -7 . This is not surprising as the mass loss rates for these stars are very large. Using the formula of Jura (1987), which relates $\mathrm{M}$ to the $60 \mu \mathrm{m}$ IRAS flux $\mathrm{F}_{60}$, and assuming an LMC distance modulus of 18.5 and a typical value of $\mathrm{F}_{60} / \mathrm{F}_{25}=0.25$, a mass loss rate of $\sim 6 \times 10^{-5} \mathrm{M}_{\mathrm{O}} \mathrm{yr}^{-1}$ can be derived for the AGB IRAS sources. The lifetimes for such stars must be less than $\sim 10^{5}$ years (the envelope masses must be no more than a few solar masses and would be dissipated by the 
large mass loss rates in $<10^{5}$ years). Since AGB stars evolve in luminosity by only 0.1 magnitude in $10^{5}$ years, the evolution of the IRAS sources in Fig 1 must be almost horizontal on the HR diagram. This also implies that there is little chance that these stars will have sufficient time on the AGB to increase their core masses to the Chandrasekhar limiting mass (which corresponds to a luminosity $\mathrm{M}_{\mathrm{bol}} \approx-7.1$ ) and become supernovae.

A final question that remains to be answered is: why do the periods of the upper AGB LPVs increase so dramatically from $\sim 600$ to $\sim 1500$ days? There are two reasons for this. The period of an LPV pulsating in the fundamental mode is given roughly by $P \propto R^{2} / M$. The first factor to cause an increase in period is the reduction in mass due to mass loss; this can be considerable in these relatively massive AGB stars. The second factor is the decrease in $\mathrm{T}_{\text {eff }}$ (increase in radius) that occurs in an AGB star when the (envelope) mass is reduced, at least until the envelope mass becomes so small that the star begins to shrink again. The formulae in Wood (1989) give quantitative estimates of these effects. These formulae can also be used to put lines of constant pulsation mass on the $\left(\mathrm{M}_{\mathrm{bol}}, \log \mathrm{P}\right)$ plane and such lines are shown in Fig 1.

A notable feature shown in Fig 1 is the gap between the optically visible LPVs with $\mathrm{M}_{\mathrm{bol}}=-6$ to -7 and their more evolved counterparts, the IRAS sources. Since the rate of evolution should speed up in going from the optically visible stars to the IRAS sources (due to the ever increasing mass loss rate), and given that there are five IRAS sources, one would expect the interval between the two groups to be well populated. The absence of stars in the gap is almost certainly a selection effect. The five AGB IRAS sources have $\mathrm{F}_{25}>0.7 \mathrm{Jy}\left(\mathrm{F}_{12}>0.5 \mathrm{Jy}\right)$, this being the selection limit of Wood et al.(1990). However, the three reddest optically visible upper AGB stars in Fig 1 were also detected by IRAS and they have $\mathrm{F}_{12} \sim 0.2 \mathrm{Jy}$, near the faint flux limit of the IRAS point source catalog. It is likely that there are other dusty, upper AGB LMC stars yet to be identified in the IRAS point source catalog. Detailed analyses of IRAS data pertaining to the Magellanic Clouds have been made by Schwering (1988) and Reid et al. (1990), and these two studies list further point sources not present in the IRAS point source catalog.

\section{Dependence of LPV period on metal abundance}

From theoretical considerations, one might expect the period of an LPV to depend on the metal abundance of the star. Since the giant branch becomes cooler at a given luminosity as metal abundance is increased, the resulting increase in radius, combined with the fact that $P \sim R^{2}$, should cause the period to increase with metallicity. Or, in terms of period luminosity (PL) relations, the luminosity at a given period should be lower in objects of higher metallicity (unless there is some counteracting effect due to the mass-period relation being different in systems of different metallicity). The LPVs in the LMC and SMC (and Baade's window) provide an opportunity to test these theoretical expectations observationally.

Fig 2 shows the $\left(\mathrm{M}_{\mathrm{bol}}, \log \mathrm{P}\right)$ diagram for AGB stars in the SMC, LMC and Baade's window, plotted assuming distance modulii of $18.9,18.5$ and 14.65 , respectively. Firstly, we examine the upper AGB stars with $M_{b o l}<-6$. The group of SMC LPVs satisfying this criterion have periods about 0.1 shorter in $\log \mathrm{P}$ than the corresponding group of LMC stars. The stars being considered here are the upper AGB stars that have not yet lost a significant amount of mass, and it will be assumed that the mean mass is similar in the SMC and LMC. The theoretical relations in Wood (1989) give, for upper AGB stars, $\log \mathrm{P}($ days $)=-3.20+0.42 \log \mathrm{Z} / \mathrm{Z}_{0}-1.61 \log \mathrm{M} / \mathrm{M}_{\mathrm{O}}+1.57 \log \mathrm{L} / \mathrm{L}_{0}$, where $\mathrm{Z}$ is the metal abundance. For young stars in the field of the SMC and LMC, Russell and 


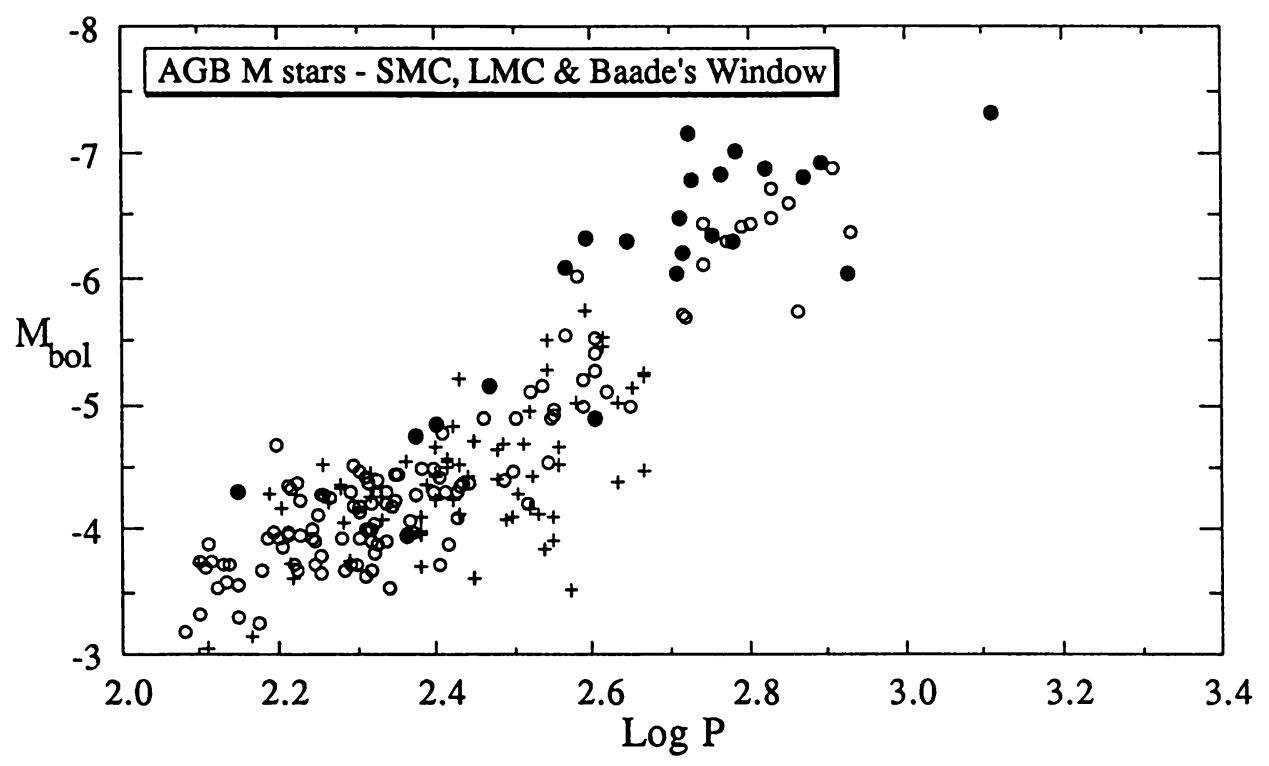

Figure 2. Mean values of $\mathrm{M}_{\mathrm{bol}}$ for optically visible LPVs with well-determined periods in the SMC (filled circles), LMC (open circles) and Baade's window (crosses). $\mathbf{M}_{\text {bol }}$ was calculated from JHK photometry listed in the text for the SMC and LMC, and from Glass and Feast (1982a), Wood and Bessell (1983) and Frogel and Whitford (1987) for Baade's window. Many objects have few JHK observations so a large part of the scatter in $\mathrm{M}_{\mathrm{bol}}$ is due to lack of good mean $\mathrm{M}_{\mathrm{bol}}$ values (see Feast et al. 1989).

Bessell (1989) and Russell and Dopita (1990) give $Z_{L M C}=Z_{\delta} / 2$ and $Z_{S M C}=Z_{O} / 4$. With these metal abundances, the above equations predict that, on the upper AGB, <logP>SMC $=\langle\log P\rangle_{L M C}-0.13$, in reasonable agreement with the period separation shown in Fig 2 . This result suggests that the periods of LPVs do indeed depend on metallicity.

The evidence for a metal dependence of the PL relation for lower luminosity AGB LPVs (Mira variables) is much more difficult to find. In the SMC, nearly all the Mira variables are carbon stars (Lloyd Evans et al. 1988) and it is difficult to find a significant number of stars to compare with the LMC M-type Miras. In Baade's window, there are significant depth effects due to stars behind, and in front of, the Galactic centre. In Fig 2, there is marginal evidence that the few M type SMC Miras lie above the LMC Miras in the mean, but multiphase observations of these and other SMC stars are required before this effect can be confirmed. There also seems to be a group of Baade's window objects with unusually low luminosity, as would be expected if these objects belong to the group of high metallicity stars near the Galactic centre discussed by Whitford and Rich (1983). However, an examination of the J-K colours of these LPVs does not indicate that they are unusually cool, and so the reason for the low apparent luminosity of these stars is probably that they lie well behind the Galactic centre.

\section{The period-luminosity-colour relation and its origin}

As mentioned above, one reason that a range of periods can exist for Miras of a given luminosity on the $A G B$ is that the giant branch $T_{\text {eff varies with metal abundance. Under }}$ these circumstances, one might expect that a period-luminosity-colour (PLC) relation 
would give a better estimate of the period of a Mira variable than would the more simple PL relation.

By obtaining mean luminosities and colours from multi-phase observations of a group of LMC Mira variables, Feast et al. (1989) were able to show that these stars do indeed obey a PLC relation. Feast $e t$ al. noted that the Miras obey both an ( $\left.\mathrm{M}_{\mathrm{bol}}, \log \mathrm{P}\right)$ relation and $a(\mathrm{~J}-\mathrm{K}, \log \mathrm{P})$ relation and they found that the deviations of individual M-type Miras from these relations were correlated in the sense that the redder stars were fainter at a given period. If $\delta \mathrm{M}_{\mathrm{bol}}$ is the deviation of an individual Mira from the mean $\mathrm{LMC}\left(\mathrm{M}_{\mathrm{bol}}\right.$, $\log \mathrm{P})$ relation and $\delta(\mathrm{J}-\mathrm{K})$ is the corresponding deviation from the $(\mathrm{J}-\mathrm{K}, \log \mathrm{P})$ relation, then Feast et al. find $\delta \mathrm{M}_{\text {bol }} \approx 2 \delta(\mathrm{J}-\mathrm{K})$, with $\delta \mathrm{M}_{\text {bol }}$ values up to $\pm 0.3 \mathrm{mag}$. and $\delta(\mathrm{J}-\mathrm{K})$ values up to \pm 0.15 magnitudes.

These results will now be compared with theory. Wood (1989) gives the following relations for Miras with $\mathrm{M}<1.5 \mathrm{M}_{0}$ :

(i) the period-mass-radius relation:

$\log \mathrm{P}=-2.07+1.94 \log \mathrm{R} / \mathrm{Rs}-0.9 \log \mathrm{M} / \mathrm{M}_{\mathrm{O}}$

(ii) the definition of $\mathrm{T}_{\text {eff }}$ :

$\mathrm{L}=4 \pi \sigma \mathrm{R}^{2} \mathrm{~T}_{\text {eff }}{ }^{4}$

(iii) the position of the AGB:

$$
\mathrm{M}_{\text {bol }}=15.7\left(\log \mathrm{T}_{\text {eff }}+0.12 \log \mathrm{Z} / \mathrm{Z}_{\mathrm{O}}-\Delta\right)-2.65 \log \mathrm{M} / \mathrm{M}_{\mathrm{O}}-59.1 \text {. }
$$

The quantity $\Delta$ accounts for the fact that, for small envelope masses, the AGB turns back to higher effective temperatures. In addition to the above equations, a relation between $\mathrm{J}-\mathrm{K}$ and $\mathrm{T}_{\text {eff }}$ is needed in the present context and for this we use the relation given by Bessell, Wood and Lloyd Evans (1983). For a typical LMC Mira with J-K $\approx 1.3\left(\mathrm{~T}_{\mathrm{eff}} \approx\right.$ $3240 \mathrm{~K})$ this relation gives

$$
\log \mathrm{T}_{\text {eff }}=3.77-0.20(\mathrm{~J}-\mathrm{K}) \text {. }
$$

Combining Equations (1), (2) and (3) gives

$$
\mathrm{M}_{\text {bol }}=-0.36-1.57 \log \mathrm{P}+0.73 \log \mathrm{Z} / \mathrm{Z}_{\mathrm{O}}-2.45 \log \mathrm{M}_{\mathrm{M}} \mathrm{M}_{\mathrm{O}}-6.1 \Delta \text {, }
$$

while combining Equations (1), (2), (3) and (4) gives

$$
\mathrm{J}-\mathrm{K}=0.15+0.50 \log \mathrm{P}+0.36 \log \mathrm{Z} / \mathrm{Z}_{\mathrm{O}}-0.065 \log \mathrm{M} / \mathrm{M}_{\mathrm{O}}-3.05 \Delta \text {. }
$$

From Equations (5) and (6), it can be seen that the scatter in $M_{b o l}$ and J-K at a given period can be due to either a scatter in $Z$ or a scatter in $M$ ( $\Delta$ is a function of $M$ and $\left.M_{b o l}\right)$. (Another possibility would be variable foreground or circumstellar extinction: this has been discussed by Feast et al. 1989 and considered unlikely.) It can be seen that a scatter in $\mathrm{Z}$ will give a scatter in $\mathrm{M}_{\mathrm{bol}}$ and $\mathrm{J}-\mathrm{K}$ which obeys the required relation $\delta\left(\mathrm{M}_{\mathrm{bol}}\right) \approx 2$ $\delta(\mathrm{J}-\mathrm{K})$; the amplitude of the observed scatter $\left(\delta \mathrm{M}_{\mathrm{bol}}<0.3\right)$ requires a scatter in the abundance among field Miras in the LMC of $\delta \log Z<0.41$. (We note here that a possible dependence of the $\left(\mathrm{M}_{\mathrm{bol}}, \log \mathrm{P}\right)$ relation for Miras on metal abundance, as predicted by Equation (5), has been observed in globular clusters by Menzies \& Whitelock (1985), although the effect is uncertain due to uncertainties in the distances to globular clusters.)

The other possible source of scatter about the $\left(\mathrm{M}_{\mathrm{bol}}, \log \mathrm{P}\right)$ and $(\mathrm{J}-\mathrm{K}, \log \mathrm{P})$ relations is a scatter in mass. If the term in $\Delta$ in Equations (5) and (6) is neglected, it is clear that a scatter in mass can not account for the observed scatter. This is because $\mathrm{J}-\mathrm{K}$ is 
to $\mathrm{M}$, and a scatter of $\sim 0.15$ in $\mathrm{J}-\mathrm{K}$, as observed, would require a scatter in $\log \mathrm{M}$ of $\sim 2.3$, clearly unrealistic. We now examine the effect of $\Delta$. If the Miras examined by Feast $e t$ al. (1989) have small envelope masses, the $\Delta$ term in Equations (5) and (6) becomes important. The $\mathrm{M}$ stars studied by Feast $e t$ al. tend to be mostly of short period and low luminosity, $M_{b o l} \approx-4.25, \log P \approx 2.4$. For $\Delta=0$ and $Z / Z_{0}=1 / 4$ (half the metal abundance of present LMC gas is assumed for these old stars), Equation (5) gives a mass of $0.74 \mathrm{Ms}$, while the luminosity-core mass relation for AGB stars (Wood \& Zarro 1981) gives a core mass of $0.56 \mathrm{M}_{0}$; the envelope mass is then $0.18 \mathrm{M}_{0}$. If mass loss removes mass from the envelopes of these stars, the star will begin to turn to bluer temperatures due to lack of envelope mass and the $\Delta$ term will become important. The equation in Wood (1989) for $\Delta$ shows that halving the envelope mass at constant $\mathrm{M}_{\text {bol }}$ from $0.18 \mathrm{M}_{0}$ to $0.09 \mathrm{M}_{0}$ increases $\log \mathrm{P}$ by 0.03 , so that the star lies fainter than the mean $\left(\mathrm{M}_{\text {bol }}, \log \mathrm{P}\right)$ relation by $\delta \mathrm{M}_{\mathrm{bol}} \approx 0.09$ magnitudes. At the same time, the star moves a distance $\delta(\mathrm{J}-\mathrm{K})$ $\approx-0.04$ mag. bluer than the mean $(\mathrm{J}-\mathrm{K}, \log \mathrm{P})$ relation. Thus $\delta\left(\mathrm{M}_{\mathrm{bol}}\right) \approx-2.25 \delta(\mathrm{J}-\mathrm{K})$, a relation very different from the observed one. This is basically because, for these modest envelope masses, the term in $\Delta$ dominates in Equation (6) while the term in log M/Mo dominates in Equation (5). As the envelope mass is reduced further, the $\Delta$ term will dominate in both Equations and the observed relation $\delta\left(\mathrm{M}_{\mathrm{bol}}\right) \approx 2 \delta(\mathrm{J}-\mathrm{K})$ will be obtained. However, for an ensemble of stars with a range of envelope masses, it is very unlikely that the observed correlation between $\delta\left(\mathrm{M}_{\mathrm{bol}}\right)$ and $\delta(\mathrm{J}-\mathrm{K})$ would be obtained from variations in envelope mass. In this situation, the most likely explanation for the scatter of the Mira-like LPVs about the $\left(\mathrm{M}_{\mathrm{bol}}, \log \mathrm{P}\right)$ and $(\mathrm{J}-\mathrm{K}, \log \mathrm{P})$ relations is a variation in metal abundance whose size is $\delta \log Z<0.4$; this is approximately a $2-\sigma$ limit since it represents the maximum deviation shown by the 29 oxygen-rich Miras studied by Feast et al. (1989). Whether this abundance variation is due to variations in the chemical content of the stars at the time of formation or due to variations caused by the first, second and third dredge-up episodes on the giant branch can not be determined.

\section{Period distributions and lifetimes}

The reasonably complete surveys that have been done for LPVs in the LMC allow a comparison of the LMC and Galactic LPV populations. Such a comparison is made by Hughes \& Wood (1990) who show that the LMC period distribution is shifted to shorter periods than the Galactic distribution. There are also many more carbon stars among the LMC LPVs, at least for $200<\mathrm{P}$ (days) $<500$. The survey data for the SMC has not yet been fully analysed, but the results of Lloyd Evans et al. (1988) \& Moore (1990) indicate that the SMC has few Mira-type variables with $\mathrm{P}<250$ days, that nearly all the Miras with $250<\mathrm{P}$ (days) $<500$ are carbon stars, and that, compared with the LMC, there is a relatively large number of upper AGB LPVs with $P>500$ days and $M_{b o l}<-6$. The bright LPVs presumably come from recent ( $10^{8}$ years ago) star formation, while the absence of shorter period LPVs with $\mathrm{P}<250$ days indicates that the $S M C$ field had $[\mathrm{Fe} / \mathrm{H}]<-1.5$ when stars that might have turned into LPVs with $\mathrm{P}<250$ days formed; the population of LPVs in globular clusters shows that $[\mathrm{Fe} / \mathrm{H}]>-1.5$ is required if a star is to pulsate as a large-amplitude LPV (Menzies \& Whitelock 1985).

The population of LPVs in the LMC allows the determination of the lifetime of typical Mira variables in the LMC. By comparing the number of Miras with the number of planetary nebulae and the number of core-helium burning clump stars, Hughes \& Wood (1990) derived a Mira lifetime of $\sim 5 \times 10^{4}$ years. This is a remarkably short lifetime, corresponding to an increase in quiescent (inter shell flash) luminosity on the AGB of only 0.05 magnitudes. In fact, the Mira lifetime is very similar to the interval between helium shell flashes and this near-equality of timescales hints that helium shell flashes may be closely related to the termination of AGB evolution. 


\section{References}

Bessell, M.S., Freeman, K.C., Wood, P.R. (1986), Ap.J. 310, 710.

Bessell, M.S., Wood, P.R., Lloyd Evans, T. (1983), M.N.R.A.S. 202, 59.

Butler, C.J. (1972), IAU Colloq.15, New Directions and New Frontiers in Variable Star Research, Veröff. Remeis-Sternw. Bamberg, 9, 90.

Butler, C.J. (1978), A\&A. Suppl. 32, 83.

Catchpole, R.M., Feast, R.M. (1981), M.N.R.A.S. 197, 385.

Dessy, J.L. (1959), Bol. Inst. Mat. Astr. Fis. Cordoba 1, no. 2.

Elias, J.H. Frogel, J.A., Humphreys, R.M. (1985), Ap.J. Suppl. 57, 91.

Feast, M.W., Catchpole, R.M., Carter, B.S., Roberts, G. (1980), M.N.R.A.S. 193, 377.

Feast, M.W., Glass, I.S., Whitelock, P.A., Catchpole, R.M. (1989), M.N.R.A.S. 241, 375.

Frogel, J.A. (1983), Ap.J. 272, 116.

Frogel, J.A., Whitford, A.E. (1987), Ap.J. 320, 199.

Glass, I.S. (1979), M.N.R.A.S. 186, 317.

Glass, I.S., Feast, M.W. (1982a), M.N.R.A.S. 198, 199.

Glass, I.S., Feast, M.W. (1982b), M.N.R.A.S. 199, 245.

Glass, I.S., Lloyd Evans, T. (1981), Nature 291, 303.

Glass, I.S., Reid, N. (1985), M.N.R.A.S. 214, 405.

Hodge, P.W., Wright, F.W. (1969), Ap.J. Suppl. 17, 467.

Hughes, S.M.G. (1989), A.J.97, 1634.

Hughes, S.M.G., Wood, P.R. (1990), A.J. 99, 784.

Jura, M. (1987), Ap.J. 313, 743.

Lloyd Evans, T. (1971), The Magellanic Clouds, A.B. Muller (ed.), (Reidel: Dordrecht), p.74.

Lloyd Evans, T. (1978), M.N.R.A.S. 183, 305.

Lloyd Evans, T. (1985), M.N.R.A.S. 212, 955.

Lloyd Evans, T., Glass, I.S., Catchpole, R.M. (1988), M.N.R.A.S. 231, 773.

Menzies, J.W., Whitelock, P.A. (1985), M.N.R.A.S. 212, 783.

Moore, G. (1990), in preparation.

Payne-Gaposhkin, C.H. (1971), Smithsonian Contr. Ap. 13, 1.

Payne-Gaposhkin, C., Gaposhkin, S. (1966), Smithsonian Contr. Ap. 9, 1.

Reid, N., Glass, I.S., Catchpole, R.M. (1988), M.N.R.A.S. 232, 53.

Reid, N., Tinney, C., Mould, J.R. (1990), Ap.J. 348, 98.

Russell, S.C., Bessell, M.S. (1989), Ap.J. Suppl. 70, 865.

Russell, S.C., Dopita, M.A. (1990), Ap.J. Suppl. in press.

Schwering, P. (1988), Ph.D. Thesis, Leiden University.

Whitelock, P.A., Feast, M.W., Menzies, J.W., Catchpole, R.M. (1989), M.N.R.A.S., 238, 769.

Whitford, A.E., Rich, R.M. (1983), Ap.J. 274, 723.

Wood, P.R. (1989), From Miras to Planetary Nebulae: Which Path for Stellar Evolution, M.O. Mennessier (ed.) (Editions Frontières), in press.

Wood, P.R., Bessell, M.S. (1983), Ap.J. 265, 748.

Wood, P.R., Bessell, M.S. (1985), Publ. Astr. Soc. Pacific 97, 681.

Wood, P.R., Bessell, M.S., Fox, M.W. (1983), Ap.J. 272, 99.

Wood, P.R., Bessell, M.S., Hughes, S.M.G., Hyland, A.R., Whiteoak, J.B., Gardner, F.F., Otrupcek, R.E. (1990), this volume.

Wood, P.R., Bessell, M.S., Paltoglou, G. (1985), Ap.J. 290, 477.

Wood, P.R., Zarro, D.M. (1981), Ap.J. 247, 247. 